\title{
ORIGINAL WORK
}

\section{Prognostic Value of Serially Estimated Serum Procalcitonin Levels in Traumatic Brain Injury Patients With or Without Extra Cranial Injury on Early In-hospital Mortality: A Longitudinal Observational Study}

\author{
Keshav Goyal ${ }^{1 *+} \mathbb{D}$, Gaurav Singh Tomar ${ }^{1 \dagger}$, Kangana Sengar $^{2+}$, Gyaninder Pal Singh ${ }^{1}$, Richa Aggarwal ${ }^{3}$,
} Kapil Dev Soni ${ }^{3}$, Purva Mathur ${ }^{4}$, Shweta Kedia ${ }^{5}$ and Hemanshu Prabhakar ${ }^{1}$

\begin{abstract}
Background: Traumatic brain injury (TBI) is associated with majority of trauma deaths, and objective tools are required to understand the severity of injury. The application of a biomarker like procalcitonin (PCT) in TBI may allow for assessment of severity and thus aid in prognostication and correlation with mortality and outcome.

Aims: The primary objective is to determine the correlation between PCT concentrations with TBI outcomes (mainly in terms of mortality) at intensive care unit (ICU)/hospital discharge. Secondary objectives are to evaluate correlation with associated extra cranial injuries and complications during hospital stay.

Methods: In total, $186 \mathrm{TBI}$ patients aged $>18$ years with minimum survival for at least $12 \mathrm{~h}$ admitted to the ICU at the level 1 trauma center were prospectively included in the study and divided into two groups: TBI with and without extra cranial injuries. All admitted patients were treated according to the standard institutional protocol. The PCT levels were obtained on admission, on day 2, and 5. Clinical, laboratory, diagnostic, and therapeutic data were also collected. Primary mortality is defined as death related to central nervous system (CNS) injury, while secondary mortality defined as death related to sepsis or extracranial cause.

Results: Median PCT levels at admission, day 2, and day 5 in TBI patients with extracranial injuries were 3.0, 0.83, and $0.69 \mathrm{ng} / \mathrm{ml}$. In total, primary mortality was observed in 18 (9.7\%) patients, while secondary causes were attributable in 20 (12.3\%) patients. Regression analysis for primarily CNS cause of mortality showed PCT cutoff level at admission more than $5.5 \mathrm{ng} / \mathrm{ml}$ carried sensitivity and specificity of 75\%, but for secondary cause (sepsis) of mortality, PCT cutoff values on day $2>1.15 \mathrm{ng} / \mathrm{ml}$ were derived significant with sensitivity of $70 \%$ and specificity of $66 \%$. No significant association of parameters like length of ICU stay, Glasgow outcome scale (GOS), and primary/secondary mortality with the presence of extracranial injuries in TBI patients as compared with TBI alone was noted.
\end{abstract}

\footnotetext{
*Correspondence: keshavgoyalster@gmail.com

†Keshav Goyal, Gaurav Singh Tomar, and Kangana Sengar have contributed equally.

${ }^{1}$ Department of Neuroanaesthesiology and Critical Care, Neurosciences Centre, All India Institute of Medical Sciences, New Delhi, 7th Floor, 710, New Delhi 110029, India

Full list of author information is available at the end of the article
} 
Conclusion: This observational study demonstrates the poor correlation between PCT concentrations with outcome at days 1,2, and 5 post-injury. The predicted relationship between PCT levels and outcome was not confirmed, and that these results do not support the prognostic utility of PCT biomarker in this population for outcome (mortality) assessment in TBI patients with or without extracranial injuries.

Keywords: Procalcitonin, Mortality, Traumatic brain injury, Sepsis

\section{Introduction}

Traumatic brain injury (TBI) patients are prone to develop fatal complications because of their primary pathology and are at risk of secondary complications such as aspiration pneumonitis, ventilator-associated pneumonia (VAP), and sepsis. Prompt diagnosis and management are necessary to reduce morbidity and mortality [1]. In normal and healthy individuals, procalcitonin (PCT) is produced in thyroid cells. Calcitonin when activated is involved in calcium and phosphorus homeostasis. Virtually all PCT is converted to calcitonin in healthy subjects so that the circulating level of PCT is $<0.05 \mathrm{ng} / \mathrm{ml}$. With inflammation, PCT is produced by a direct lipopolysaccharide (or other toxic metabolite from microbes) pathway and an indirect pathway from inflammatory mediators [2]. This has been demonstrated in previous studies that PCT levels are found to be raised in patients with bacterial sepsis, systemic inflammatory response syndrome (SIRS), and also after traumatic injury therefore can aid in prompt diagnosis [3-6]. PCT levels may be a better indicator in the early diagnosis of septic complications in patients with multiple trauma [3]. However, during the first 2 days after trauma elevated PCT levels are more likely to be indicative of traumatic origin than of an ongoing sepsis, and multiple events such as surgery, massive transfusion, intensive care therapy might influence the PCT concentration [7]. TBI is associated with the majority of trauma deaths [8]. Clinicians are often confronted with questions related to the severity of injury and prognosis. Predicting outcome becomes difficult due to the heterogeneity of TBI, some patients recover well, while others are left with severe neurological disability. Objective tools are required to understand the severity of injury and then these tools can be useful to predict the prognosis. The application of a biomarker like PCT for TBI could lead to assess the severity and thus help in prognostication and correlation with mortality and outcome. We hypothesize that greater severity of TBI and/ or associated extracranial injuries is more often encountered with higher PCT concentration and poor outcomes.

PCT has been seen as the prognostic marker in multiple traumatic injury patients, but its comparative role has not been assessed as a prognostic marker based on outcomes in different types of TBI [3-6]. This study was planned to determine outcome in different groups of TBI patients with and without extracranial injury as assessed by serial evaluation of PCT.

\section{Objectives}

Primary objective is to determine the correlation between PCT concentrations with TBI outcomes (primarily, inhospital mortality) at neurointensive care unit (ICU)/ hospital discharge assessed using Glasgow outcome scale (GOS). The secondary objectives are to evaluate for any correlation with associated extra cranial injuries and complications during hospital stay.

\section{Materials and Methods}

After approval from institutional ethics committee (ref. no. IEC/NP-391/2012 \& RP-43/2012), all patients with TBI admitted to the ICU at the level 1 apex trauma center unit were prospectively included in the study. This study has been registered prospectively with clinical trial registry of India (CTRI/05/008466). The patients were divided into two major groups: head injury with extracranial injury or without extracranial injury. The inclusion criteria were patients with head injury aged $>18$ years, and survival for at least $12 \mathrm{~h}$ after admission admitted to the study center within $8 \mathrm{~h}$ of injury and patients with positive head injury findings on computer tomography (CT) scan. Exclusion criteria included patients with preexisting sepsis or with chronic kidney disease on regular dialysis requirement, patients with history of drug abuse or any anaphylaxis. Patients were treated according to the institutional protocol and accepted standards of care. PCT, all laboratory, microbiological, and clinical data with duration of treatment in the ICU/hospital were noted. PCT values were obtained on day 1,2 , and 5 after trauma. The PCT levels were measured by the mini VIDAS ${ }^{\circledR}$ assay, Biomerieux Pvt. Ltd., i.e., based on the enzyme-linked fluorescent assay principal. The PCT level more than $0.5 \mathrm{ng} / \mathrm{ml}$ was taken as positive and greater than $10 \mathrm{ng} / \mathrm{ml}$ as highly significant. The data collected during admission were age, gender, mode of injury (road traffic accident, fall, or assault), chronic conditions if any, GCS, severity of trauma according to ISS (injury severity score) [9], and number of blood units transfused within first $24 \mathrm{~h}$ after trauma. Complications included were suspected pneumonia, SIRS, sepsis, or septic shock and the occurrence of organ dysfunction (respiratory, 
cardiac, renal, or hepatic). The amount of blood loss was also noted. The final data analyzed were survival data at discharge from the ICU and hospital. Intravenous (IV) broad spectrum antibiotics were administered to all ICUadmitted patients in accordance with ICU and hospital protocol.

Brain CT scans of TBI patients were rated by using the Marshall grading scale [10]. After primary diagnostic assessment and/or surgery, all patients were transferred to the ICU and received standard intensive care, including intubation and mechanical ventilation, hemodynamic and intracranial pressure (ICP) monitoring using Codman microsensor ${ }^{\mathrm{TM}}$ based on the severity of injury and institutional protocol. Lastly, 1 month after the primary injury, the study protocol was documented for outcome according to the survival and GOS scoring 1-5 (1, dead; 2 , persistent vegetative state; 3 , severely disabled; 4 , moderately disabled; 5, good recovery). The probable etiology of mortality was also noted. The primary mortality was defined as loss of life because of severity of traumainduced CNS pathology in TBI patients, whereas secondary mortality as mortality because of complications in TBI patients such as sepsis, VAP, coagulopathy, or associated injuries. Final outcome on discharge was classified on basis of GOS as good outcome (scoring 4-5) or poor outcome (score of 2-3).

\section{Statistical Analysis}

Nonparametric continuous variables are expressed as the median and inter-quartile ranges, and normally distributed variables are presented as the mean \pm standard deviation (SD). Statistical data were analyzed using Stata Corp. 2015. Stata Statistical Software: (Release 14) College Station, Texas. PCT levels among TBI patients with and without extracranial injuries groups were carried out using Kruskal-Wallis tests (data not normally distributed), and if statistical significance of differences was detected, then the two-sample Wilcoxon rank-sum (Mann-Whitney) (nonparametric analysis) was used for further comparisons between the two comparison groups. We used the Pearson Chi-square test ( $\chi^{2}$ test) and Fisher's exact test to compare proportions. Univariate and multivariate logistic regression with odds ratio was used to assess the performance of the variables in the prediction of primary or secondary outcome. The sample of the present study was a result of the inclusion of patients admitted to ICU of a level 1 trauma center in a 2 -year period. Thus, a post hoc analysis was performed to determine the power of the study calculated out to be nearly $80 \%$ for the primary outcome.

\section{Results}

A total of 188 patients were screened for this study out of those 2 were excluded ( 1 refused consent and other did not meet criteria for inclusion) in accordance with STROBE flow chart of patient's recruitment (Fig. 1). Hence, 186 patients were included in the study between January 2014 and January 2016 for the first wave of data collection followed by 163 patients for the second wave and finally 110 for the last wave of data collection (Fig. 1). Table 1 compares the baseline demographic and various clinical characteristics between patients. The majority of patients presented were found to have intracranial pathologies like subdural hematoma (SDH), extradural hematoma (EDH), contusions, subarachnoid hemorrhage (SAH), intraventricular hemorrhage (IVH), and diffuse axonal injuries (DAI) (Table 2). Twenty-eight TBI patients were found to have associated extracranial injuries or positive FAST (Focused Assessment Sonography in Trauma) scan findings. The median PCT levels in severe TBI patients with GCS [3-5] were found to be consistently higher (3.37 on day $1,2.1$ on day 2 , and 1.25 on day 5 ) at all time than patients with GCS in range 6-8; or 9-12 (Table 3). Among them, long bones (femur/ radius/ulna/tibia/fibula) or pelvis fractures were noted in 14 patients, maxillo-facial fractures in 11 patients, lung contusions (unilateral/bilateral) in 10 patients, $C_{1}-C_{2}$ spine subluxation in 6 patients, splenic tear/laceration in 8 patients, liver contusions/lacerations in 7 patients, hemo-pneumothorax in 5 patients, bilateral/unilateral lung contusions in 4 patients, and intestinal tear/laceration in 3 patients. We were able to get ICP monitoring in only 17 patients (out of total 21 DAI patients). In rest other cases/indications, serial clinical CNS examinations (decreased GCS/power/abnormal pupil size/reactivity) and regular hemodynamic monitoring (sudden perturbance in HR, BP) were the tip to proceed with decompressive craniectomy. Thirty-seven patients (22.7\%) among the 163 studied patients met with sepsis/bacteremia at any point of hospital or ICU stay; 20 patients died among them from other than primary CNS cause of mortality, among them, 2 died from VAP or acute respiratory distress syndrome (ARDS) and 1 patient from septic shock. The median PCT levels were 2.49 (range 0.05, 59.1 ) and 3.36 (range $0.06,24.1$ ) $\mathrm{ng} / \mathrm{ml}$ on day 2 and 5 , respectively, among septic TBI patients, i.e., found to be highly significant compared to PCT in other TBI patients (Table 4). Among all factors observed, only presence of $\mathrm{SDH}$, presence of dilated pupils, coagulopathy, hemodynamic instability, and poor GCS at admission were found to have statistically significant association with primary mortality $(p<0.05)$ (Table 1$)$. In this study, median PCT level at admission, day 2, and day 5 in TBI patients with extracranial injuries was 3.0, 0.83 , and $0.69 \mathrm{ng} /$ 


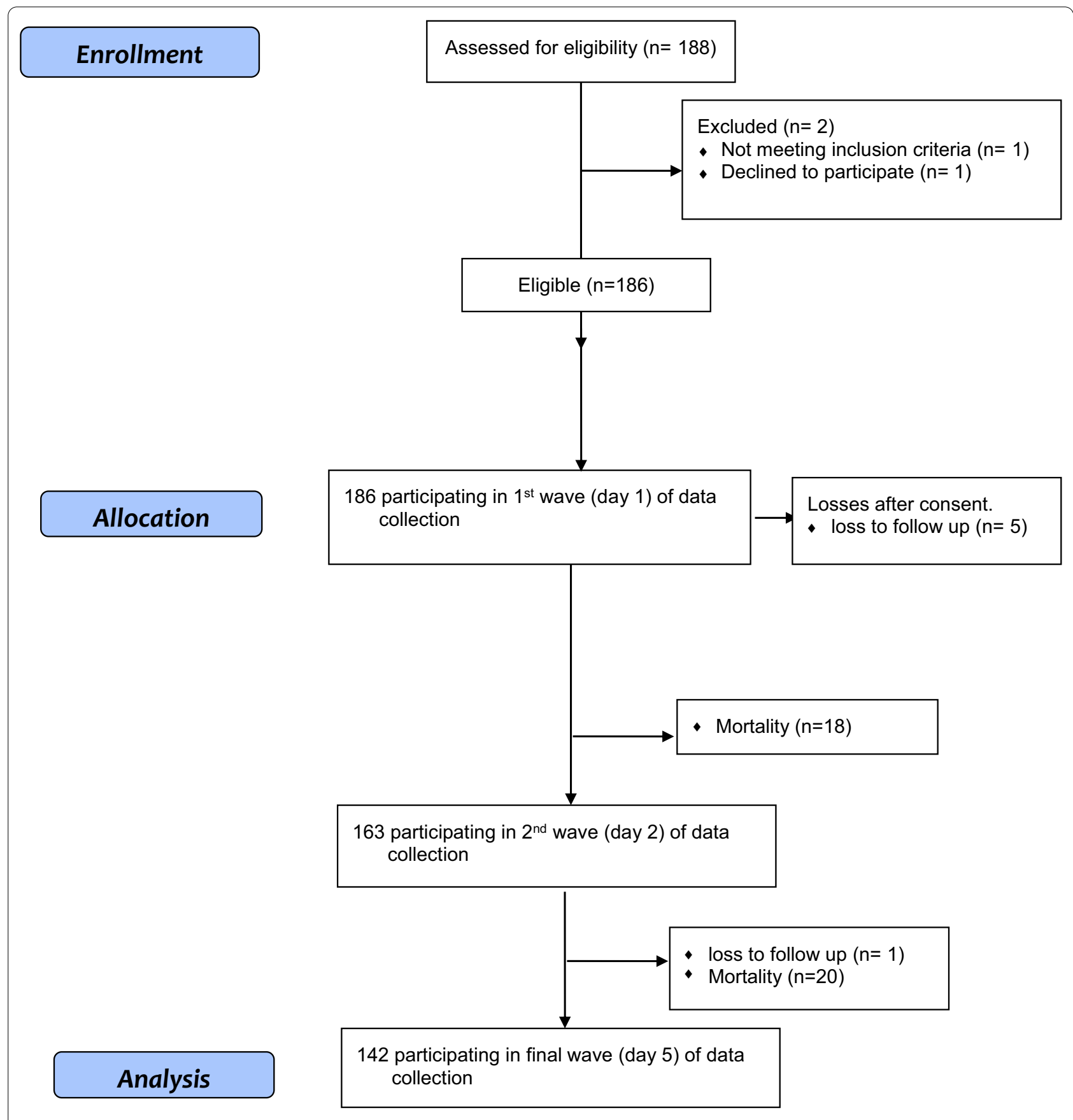

Fig. 1 STROBE flow diagram for recruitment of cases in the study

$\mathrm{ml}$. However, this was statistically found to be comparable with TBI patients without any extracranial injuries (Table 5). Considering PCT as a prediction marker of survivor or nonsurvivor, we observed median values at admission $(13.09 \mathrm{ng} / \mathrm{ml})$ and on day $2(6.37 \mathrm{ng} / \mathrm{ml})$ were significantly very high $(p \leq 0.001)$ in TBI patients those who succumbs later (Table 6). We did not observe any significant association of parameters like length of
ICU stay, GOS, and secondary mortality with the presence of extracranial injuries in TBI patients as compared to those with TBI alone ( $p$ value $>0.1$; Table 7$)$. Furthermore, through receiver operating curve (ROC) curve analysis we found that PCT levels at day 1 had a highest discriminating ability for primary mortality (AUC 78\% and cutoff level more than $5.5 \mathrm{ng} / \mathrm{ml}$ carried maximum sensitivity and specificity of 75\%) followed by PCT levels 
Table 1 Baseline demographics, clinical characteristics of TBI patients in association with primary mortality

\begin{tabular}{|c|c|c|c|}
\hline \multirow[t]{2}{*}{ Association with variables } & \multicolumn{2}{|l|}{ Primary mortality } & \multirow[t]{2}{*}{$p$ value } \\
\hline & No (survivors) freq. (\%) $\left[n_{1}=168\right]$ & Yes (died) freq. (\%) $\left[n_{2}=18\right]$ & \\
\hline Age (years) [Mean \pm SD] & $29.78 \pm 5.32$ & $32.42 \pm 5.92$ & 0.06 \\
\hline Body weight (kgs) [Mean \pm SD] & $57.72 \pm 16.4$ & $59.93 \pm 15.11$ & 0.10 \\
\hline \multicolumn{4}{|l|}{ Gender } \\
\hline Female & $25(14.7)$ & $3(18.7)$ & \\
\hline Male & $145(85.3)$ & $13(81.3)$ & 0.712 \\
\hline \multicolumn{4}{|l|}{ Mechanism of injury } \\
\hline Unknown & $3(1.7)$ & $0(0)$ & \\
\hline Road traffic accidents & $117(68.8)$ & $14(87.5)$ & \\
\hline Fall & $42(24.7)$ & $2(12.5)$ & \\
\hline Assault & $8(4.7)$ & $0(0)$ & 0.584 \\
\hline \multicolumn{4}{|l|}{ Chronic comorbidities } \\
\hline Absent & $154(95.0)$ & $15(90.9)$ & \\
\hline Present & $16(4.9)$ & $1(9.0)$ & 0.45 \\
\hline \multicolumn{4}{|l|}{ Management } \\
\hline Conservative & $74(45.6)$ & $10(54.5)$ & \\
\hline Surgical intervention & $88(54.3)$ & $6(45.4)$ & 0.756 \\
\hline \multicolumn{4}{|l|}{ Hypertension } \\
\hline Absent & $163(93.1)$ & $9(81.8)$ & \\
\hline Present & $12(6.9)$ & $2(18.1)$ & 0.148 \\
\hline \multicolumn{4}{|l|}{ Inotrope(s) requirement } \\
\hline No & $158(91.9)$ & $9(81.8)$ & \\
\hline Yes & $13(8.0)$ & $2(18.1)$ & 0.247 \\
\hline \multicolumn{4}{|c|}{ Intracranial hypertension (ICP > 20 mmHg) } \\
\hline Absent & $149(90.9)$ & $8(72.7)$ & \\
\hline Present & $14(9.1)$ & $3(27.2)$ & 0.068 \\
\hline \multicolumn{4}{|l|}{ Subdural hematoma (SDH) } \\
\hline Absent & $117(72.2)$ & $4(36.36)$ & \\
\hline Present & $45(86.5)$ & $7(13.46)$ & 0.018 \\
\hline \multicolumn{4}{|l|}{ Contusions } \\
\hline No & $97(59.8)$ & $4(36.3)$ & \\
\hline Yes & $65(40.1)$ & $7(63.6)$ & 0.204 \\
\hline \multicolumn{4}{|l|}{ Extradural hematoma (EDH) } \\
\hline No & $137(84.5)$ & $11(100)$ & \\
\hline Yes & $25(15.4)$ & 0 & 0.369 \\
\hline \multicolumn{4}{|l|}{ Diffuse axonal injury (DAl) } \\
\hline No & $143(88.2)$ & $9(81.8)$ & \\
\hline Yes & $19(11.7)$ & $2(18.1)$ & 0.626 \\
\hline \multicolumn{4}{|l|}{ Traumatic SAH/IVH } \\
\hline No & $127(78.4)$ & $7(63.6)$ & \\
\hline Yes & $35(21.6)$ & $4(36.3)$ & 0.271 \\
\hline \multicolumn{4}{|l|}{ Chest X-ray finding } \\
\hline No & $148(91.3)$ & $10(90.9)$ & \\
\hline Yes & $14(8.6)$ & $1(9.0)$ & 1.0 \\
\hline \multicolumn{4}{|l|}{ Dyselectrolytemia } \\
\hline No & $153(94.4)$ & $9(81.8)$ & \\
\hline Yes & $9(5.5)$ & $2(18.1)$ & 0.148 \\
\hline \multicolumn{4}{|l|}{ Associated injuries or (FAST + ve) } \\
\hline No & $162(93.8)$ & $9(81.8)$ & \\
\hline
\end{tabular}


Table 1 (continued)

\begin{tabular}{|c|c|c|c|}
\hline \multirow[t]{2}{*}{ Association with variables } & \multicolumn{2}{|l|}{ Primary mortality } & \multirow[t]{2}{*}{$p$ value } \\
\hline & No (survivors) freq. (\%) $\left[n_{1}=168\right]$ & Yes (died) freq. (\%) $\left[n_{2}=18\right]$ & \\
\hline Yes & $13(6.17)$ & $2(18.1)$ & 0.17 \\
\hline \multicolumn{4}{|c|}{ Dilated pupils (unilateral or bilateral) } \\
\hline No & $147(90.7)$ & $4(36.3)$ & \\
\hline Yes & $15(9.2)$ & $7(63.6)$ & 0.0001 \\
\hline \multicolumn{4}{|c|}{ Surgery: decompressive craniectomy } \\
\hline No & $108(66.6)$ & $8(72.70)$ & \\
\hline Yes & $54(33.3)$ & $3(27.2)$ & 1.00 \\
\hline \multicolumn{4}{|l|}{ Cervical-spine involvement } \\
\hline No & $149(85.8)$ & $10(90.9)$ & \\
\hline Yes & $26(14.2)$ & $1(9.0)$ & 1.00 \\
\hline \multicolumn{4}{|l|}{ Major blood loss (> 50\% of EBV) } \\
\hline No & $146(90.1)$ & $11(100)$ & \\
\hline Yes & $16(9.8)$ & $0(0)$ & 0.602 \\
\hline \multicolumn{4}{|c|}{ Prolonged duration of surgery ( $>6 \mathrm{~h}$ ) } \\
\hline No & $143(88.8)$ & $11(100)$ & \\
\hline Yes & $18(11.1)$ & $0(0)$ & 0.608 \\
\hline \multicolumn{4}{|l|}{ Blood products transfusion } \\
\hline No & $137(85.0)$ & $9(81.8)$ & \\
\hline Yes & $24(14.9)$ & $2(18.1)$ & 0.673 \\
\hline \multicolumn{4}{|l|}{ Complications: sepsis } \\
\hline No & $128(79.0)$ & $8(72.7)$ & \\
\hline Yes & $34(21.0)$ & $3(27.3)$ & 0.704 \\
\hline \multicolumn{4}{|l|}{ Coagulopathy } \\
\hline No & $159(98.1)$ & $9(81.8)$ & \\
\hline Yes & $3(1.8)$ & $2(18.2)$ & 0.03 \\
\hline \multicolumn{4}{|c|}{ Ventilator-associated pneumonia (VAP/ARDS) } \\
\hline No & $142(87.6)$ & $9(81.8)$ & \\
\hline Yes & $20(12.4)$ & $2(18.2)$ & 0.634 \\
\hline \multicolumn{4}{|c|}{ Hypotension or hypoxia at admission } \\
\hline No & $150(92.6)$ & $6(54.5)$ & \\
\hline Yes & $12(7.4)$ & $5(45.5)$ & $<0.001$ \\
\hline \multicolumn{4}{|c|}{ Polytrauma (extracranial involvement) } \\
\hline No & $149(85.1)$ & $9(81.8)$ & \\
\hline Yes & $26(14.9)$ & $2(18.2)$ & 0.40 \\
\hline \multicolumn{4}{|c|}{ Glasgow coma scale_-at admission } \\
\hline $9-12$ & $48(29.6)$ & $0(0)$ & \\
\hline $4-8$ & $26(16.0)$ & $1(9.1)$ & \\
\hline 3 & $88(54.3)$ & $10(90.9)$ & 0.04 \\
\hline \multicolumn{4}{|l|}{ Injury severity score (ISS) } \\
\hline Median (range) & $23(19,26)$ & $26(21,30)$ & 0.17 \\
\hline \multicolumn{4}{|l|}{ ICU stay duration } \\
\hline Median (range) days & $5(1,28)$ & $4.5(2,16)$ & 0.93 \\
\hline
\end{tabular}

Statistically significant numbers are given in bold

No: were patients who survived, while Yes: were patients who died

EBV estimated blood volume, FAST Focused Assessment Sonography in Trauma, ICP intracranial pressure, SAH/IVH subarachnoid or intraventricular hemorrhage

on day 2 with discriminating ability for secondary (sepsis, etc.) mortality (cutoff values more than $1.15 \mathrm{ng} / \mathrm{ml}$ had sensitivity of $70 \%$ with specificity of $66 \%$ ) (Table 8 ). Further we found that there was no significant difference 
Table 2 Radiological characteristics of patients with moderate to severe TBI on admission

\begin{tabular}{|c|c|c|}
\hline Initial CT brain findings (in majority of cases) & Marshall CT grading & $\begin{array}{l}\text { No. } \\
\text { of patients } \\
(n=186)\end{array}$ \\
\hline Nil/not significant lesions & । & 0 \\
\hline Small frontal hematoma/EDH, SDH, DAl grade (I) & $\|$ & 15 \\
\hline Traumatic diffuse SAH, cerebral edema, IVH, dot contusions, DAl grade (II-III) $[\mathrm{MLS} \leq 5 \mathrm{~mm}]$ & III & 35 \\
\hline Large FTP SDH/contusions (lesions size $<25 \mathrm{~cm}^{3}$ but with MLS $>5 \mathrm{~mm}$ ) & IV & 25 \\
\hline (lesions size $>25 \mathrm{~cm}^{3}$ with MLS> $>5 \mathrm{~mm}$ ) Operated/surgically evacuated lesions & V & 94 \\
\hline $\begin{array}{l}\text { Diffuse SAH, posterior fossa IC bleed, basifrontal/brainstem contusions, large occipital SDH (or lesions } \\
\text { size }>25 \mathrm{~cm}^{3} \text { with established brainstem infarct) }\end{array}$ & $\mathrm{Vl}$ & 17 \\
\hline
\end{tabular}

$D A /$ diffuse axonal injury, EDH extradural hematoma, $I C$ intracranial, $I V H$ intraventricular hemorrhage, $M L S$ midline shift, SAH sub-arachnoid hemorrhage, SDH subdural hematoma

Table 3 Procalcitonin levels in different subsets of TBI patients based on GCS

\begin{tabular}{lllr}
$\begin{array}{l}\text { Glasgow coma } \\
\text { scale }(\mathrm{GCS}) \\
\text { score }\end{array}$ & \multicolumn{2}{l}{ Procalcitonin levels } \\
\cline { 2 - 4 } & PCT-1 & \multicolumn{1}{c}{ PCT-2 } & \multicolumn{1}{c}{ PCT-5 } \\
\hline $3-5$ & $3.37(0.17-152.2)$ & $2.1(0.08-73.4)$ & $1.25(0.16-33.54)$ \\
$6-8$ & $0.79(0.05-145.7)$ & $0.58(0.04-65.8)$ & $1.0(0.2-50.6)$ \\
$9-12$ & $0.58(0.05-70.1)$ & $0.43(0.05-59.5)$ & $0.79(0.1-24.8)$
\end{tabular}

PCT-1, PCT-2, PCT-5 are procalcitonin levels expressed as median (range min.max.) on admission day, 2nd day, and 5th day in hospital, respectively

Table 4 Procalcitonin levels in TBI patients with and without sepsis

\begin{tabular}{llll} 
Procalcitonin & \multicolumn{2}{l}{ Sepsis in TBI patients } & $\begin{array}{l}\text { Statistical } \\
\text { levels }\end{array}$ \\
\cline { 2 - 3 } & Absent $(\boldsymbol{n}=126)$ & Present $(\boldsymbol{n}=\mathbf{3 7})$ & $\begin{array}{l}\text { Aificance }(\boldsymbol{p} \\
\text { value })\end{array}$ \\
\hline PCT-2 $(\mathrm{ng} / \mathrm{ml})$ & $0.42(0.04,145.7)$ & $2.49(0.05,59.1)$ & 0.0001 \\
PCT-5 $(\mathrm{ng} / \mathrm{ml})$ & $0.47(0.01,50.6)$ & $3.36(0.06,24.1)$ & 0.0002 \\
\hline
\end{tabular}

PCT values expressed as median (min.-max.) ng/ml

Table 5 Association of PCT rise with or without extracranial injuries in TBI patients

\begin{tabular}{llll}
$\begin{array}{l}\text { Associated } \\
\text { extracranial } \\
\text { injuries }\end{array}$ & PCT-1 $(\mathbf{n g} / \mathrm{ml})$ & PCT-2 $(\mathbf{n g} / \mathrm{ml})$ & PCT-5 $(\mathbf{n g} / \mathbf{m l})$ \\
\hline Absent $(n=158)$ & $0.97(0.05,152.1)$ & $0.56(0.04,145.7)$ & $0.83(0.0,50.6)$ \\
Present $(n=28)$ & $3.0(0.33,27.8)$ & $0.83(0.05,17.2)$ & $0.69(0.14,7.9)$ \\
( $p$ value) & 0.19 & 0.83 & 0.70 \\
\hline
\end{tabular}

Values are expressed as median (range min.-max.)

$P C T$ procalcitonin

in PCT levels at day 5. On univariate analysis, PCT (on day 1and 2) cutoff values more than $5.5 \mathrm{ng} / \mathrm{ml}$ and $1.16 \mathrm{ng} / \mathrm{ml}$ had odds ratio [OR] of 9.1 and 4.2 , respectively, for primary mortality. After adjusting for other known confounders like age, comorbidities, or infection at admission and complications (viz post-injury sepsis, VAP, associated injuries, FAST-positive status, positive $\mathrm{X}$-ray findings for aspiration pneumonitis/pneumotho$\mathrm{rax} /$ hemothorax/pleural-effusion/atelectasis, and coagulopathy) the adjusted OR was 8.2 and 6.3 on day 1 and 2 , respectively. Similarly, for secondary mortality and final outcome at discharge from hospital, PCT cutoff values at admission $(>2.07 \mathrm{ng} / \mathrm{ml})$, on day $2(>1.15 \mathrm{ng} / \mathrm{ml})$ presented with OR $(3.4 ; 3.6)$ and $(3.43 ; 4.9)$, respectively, and after adjusting for confounding factors like primary mortality, mechanism of trauma, intracranial HTN, presence of intracranial CT findings, dilated pupils, and perioperative factors (decompressive craniectomy, blood loss, duration of surgery, blood product transfusion, colloid administration) adjusted OR were $(2.5 ; 4.8)$ \& (2.9; 4.0 ), respectively (Table 9). Furthermore, we observed that presence of significant nonoperated EDH, persistent hypoxia before admission was independent risk factors for poor and fatal outcome, while intracranial hypertension and/or hypoxia was independent risk factors for prolonged ICU and hospital stay (Table 1).

\section{Discussion}

This observational longitudinal study demonstrates the relation between PCT concentrations with outcome at day 1 , day 2, and day 5 post-injury. This study also attempts to verify its correlation with extracranial injury and infectious complications in severe TBI patients.

PCT is an amino acid peptide, i.e., precursor molecule of calcitonin, without any known hormonal activity [11]. Serum levels of PCT linearly correlate with bacterial infection/sepsis in patients. The PCT biomolecule has been demonstrated and considered as a diagnostic and prognostic marker of bacterial sepsis among critically ill patients with better accuracy if integrated with clinical evaluation [12]. On the other hand, PCT has been found 
Table 6 PCT as a marker of survivors versus nonsurvivors in all TBI patients (No: were patients who survived, while Yes: were patients who died)

\begin{tabular}{|c|c|c|c|}
\hline \multirow[t]{2}{*}{ Parameter } & \multicolumn{2}{|l|}{ Primary mortality } & \multirow{2}{*}{$\begin{array}{l}\text { Statistical } \\
\text { significance ( } p \\
\text { value) }\end{array}$} \\
\hline & No (survivors) $\left(n_{1}=163\right)$ & Yes (died) $\left(n_{2}=18\right)$ & \\
\hline PCT-1 (ng/ml) & $1.06(0.05,152.2)$ & $13.09(0.17,65.8)$ & 0.006 \\
\hline PCT-2 (ng/ml) & $0.50(0.04,145.7)$ & $6.37(0.05,73.3)$ & 0.001 \\
\hline PCT-5 (ng/ml) & $0.79(0.0,50.6)$ & $1.32(0.05,33.5)$ & 0.15 \\
\hline
\end{tabular}

Statistically significant numbers are given in bold

Values are expressed as median (range min.-max.)

$P C T$ procalcitonin

Table 7 Baseline demographics, clinical characteristics in TBI patients with and without extracranial injuries

\begin{tabular}{|c|c|c|c|}
\hline $\begin{array}{l}\text { Baseline demographics and clinical character- } \\
\text { istics }\end{array}$ & $\begin{array}{l}\text { Head injury without extracranial } \\
\text { injuries }\end{array}$ & Head injury with extracranial injuries & $p$ value \\
\hline No. of patients & $158(84.9 \%)$ & $28(15.1 \%)$ & \\
\hline Age (years) (Mean $\pm S D)$ & $32.36 \pm 14.8$ & $34.83 \pm 16.0$ & 0.83 \\
\hline Body weight (kgs) (Mean \pm SD) & $58.79 \pm 15.20$ & $60.33 \pm 10.70$ & 0.92 \\
\hline Sex (M:F) ratio & $5: 1$ & $4: 1$ & 0.7 \\
\hline Injury severity score (0-75) & $22 \pm 7$ & $25 \pm 9$ & 0.05 \\
\hline ICU Stay, median (range) days & $5(1,28)$ & $5.5(1,15)$ & 0.47 \\
\hline GOS (1-5), median (range) & $3(1,5)$ & $2(1,5)$ & 0.06 \\
\hline Primary mortality (\%) & $9(5.7 \%)$ & $9(32 \%)$ & $<0.0001$ \\
\hline Secondary mortality (\%) & $18(11.4 \%)$ & $2(7.1 \%)$ & 0.36 \\
\hline
\end{tabular}

GOS Glasgow outcome scale, $M / F$ male/female ratio

Table 8 Area under curve (AUC) for the PCT cutoff values and their sensitivity or specificity

\begin{tabular}{|c|c|c|c|c|c|c|}
\hline Outcome & $\begin{array}{l}\text { PCT cutoff values } \\
\text { (ng/ml) }\end{array}$ & AUC ( $95 \%$ C.I.) & Sensitivity (\%) & Specificity (\%) & $\mathrm{LR}^{+}$ & LR- \\
\hline \multirow[t]{2}{*}{ Primary mortality } & PCT- $1 \geq 5.5$ & $0.78(0.66,0.89)$ & 75 & 75.3 & 3.03 & 0.33 \\
\hline & PCT- $2 \geq 1.16$ & $0.69(0.48,0.89)$ & 63.6 & 63.8 & 1.75 & 0.56 \\
\hline \multirow[t]{2}{*}{ Secondary mortality } & PCT-1 $\geq 2.07$ & $0.67(0.42,0.71)$ & 66.7 & 57.6 & 1.57 & 0.58 \\
\hline & PCT- $2 \geq 1.15$ & $0.65(0.52,0.81)$ & 70 & 65.7 & 2.04 & 0.45 \\
\hline Poor outcome at discharge & PCT-5 $\geq 1.19$ & $0.70(0.29,0.83)$ & 57.1 & 68.3 & 1.8 & 0.62 \\
\hline
\end{tabular}

$A U C$ area under curve, $C l$ confidence interval, $L R$ likelihood ratio, $P C T$ procalcitonin

capable to differentiate sepsis from other inflammatory causes and aids in vindicating antibiotic therapy in community-acquired pneumonia [13], lower respiratory tract infection [14], and sepsis or septic shock [15].

In the present study, patients with raised plasma PCT concentration on admission were variably associated with various complications, including the length of ICU stay and poor or fatal outcome. Additionally, this was evident from the consecutive raised PCT values in TBI patients that signified as a marker for sepsis soon after the decline in initial trauma-induced high PCT level. The PCT had shown to be of significance in TBI patients at admission and on follow-up examination for improving patient care and understanding prognosis quite early. In previous studies [16-18], polytrauma patients have been prone to develop complications such as infections and sepsis. Levels of PCT were reported to be significantly higher in cases with sepsis or septic shock compared with cases who developed SIRS [3]. In polytrauma (with abdominal injury) patients, PCT was reportedly found to be significantly increased $3.37 \pm 0.92 ; 3.27 \pm 0.97 \mathrm{ng} / \mathrm{ml}$ during post-injury day 1 and 2, respectively [7]. Higher PCT concentrations might pose patients susceptible for greater risk of secondary complications like sepsis, VAP/ARDS, or poor or fatal outcome. However, PCT rise varied according to the severity of trauma that did not correlate 
Table 9 Univariate and multivariate logistic regression analysis adjustment in outcomes among TBI patients

\begin{tabular}{|c|c|c|c|c|c|c|c|}
\hline Parameters & $\begin{array}{l}\text { PCT cutoff } \\
\text { value }(\mathrm{ng} / \mathrm{ml})\end{array}$ & $\begin{array}{l}\text { Yes (died) } \\
\text { No.(frequency \%) }\end{array}$ & $\begin{array}{l}\text { No (survivors) } \\
\text { No. (frequency\%) }\end{array}$ & $\begin{array}{l}\text { Unadjusted odds } \\
\text { ratio }(95 \% \mathrm{Cl})\end{array}$ & $p$ value & $\begin{array}{l}\text { Adjusted odds } \\
\text { ratio }(95 \% \mathrm{Cl})\end{array}$ & $p$ value \\
\hline \multirow[t]{4}{*}{ Primary mortality } & PCT-1 $<5.5$ & $4(3 \%)$ & $128(97 \%)$ & 1 & $<0.001$ & 1 & 0.003 \\
\hline & PCT-1 $\geq 5.5$ & $12(22.2 \%)$ & $42(77.8 \%)$ & $9.14(2.8,29.8)$ & & $8.21(2.04,32.9)$ & \\
\hline & PCT $-2<1.16$ & $4(4 \%)$ & $98(96 \%)$ & 1 & 0.039 & 1 & 0.023 \\
\hline & PCT-2 $\geq 1.16$ & $7(11.5 \%)$ & $54(88.5 \%)$ & $4.2(1.07,16.48)$ & & $6.37(1.28,31.58)$ & \\
\hline \multirow[t]{6}{*}{ Secondary mortality } & PCT-1<2.07 & $3(1.8 \%)$ & $93(57 \%)$ & 1 & 0.003 & 1 & 0.21 \\
\hline & PCT-1 $\geq 2.07$ & $8(4.9 \%)$ & $59(36.2 \%)$ & $3.43(1.54,7.65)$ & & $2.52(0.58,11.0)$ & \\
\hline & $\mathrm{PCT}-2<1.15$ & $7(4.3 \%)$ & 95 (58.3\%) & 1 & 0.009 & 1 & 0.01 \\
\hline & PCT-2 $\geq 1.15$ & $13(8 \%)$ & $48(29.4 \%)$ & $3.67(1.37,9.81)$ & & $4.79(1.38,16.61)$ & \\
\hline & PCT-5 $<1.19$ & $2(1.8 \%)$ & $48(43.6 \%)$ & 1 & 0.08 & 1 & 0.05 \\
\hline & PCT-5 $\geq 1.19$ & $3(2.7 \%)$ & $47(42.7 \%)$ & $1.18(1.2,3.5)$ & & $1.82(1.32,3.20)$ & \\
\hline \multirow[t]{4}{*}{ Poor outcome } & PCT-1<2.07 & $11(11 \%)$ & $59(59 \%)$ & 1 & 0.003 & 1 & 0.16 \\
\hline & PCT-1 $\geq 2.07$ & $9(9 \%)$ & $21(21 \%)$ & $3.43(1.54,7.65)$ & & $2.92(0.64,13.30)$ & \\
\hline & PCT- $2<1.15$ & $8(8 \%)$ & $32(32 \%)$ & 1 & $<0.001$ & 1 & 0.09 \\
\hline & PCT-2 $\geq 1.15$ & $7(7 \%)$ & $53(53 \%)$ & $4.96(2.0,12.22)$ & & $4.04(0.80,20.4)$ & \\
\hline
\end{tabular}

Statistically significant numbers are given in bold

No: were patients those who survived, while Yes: were patients who died

[Primary mortality adjusted for factors like age, comorbidities, or infection at admission, complications sepsis, VAP, associated injuries, FAST-positive status, positive $\mathrm{X}$-ray findings, coagulation disorders. Secondary mortality adjusted for factors like primary mortality, mechanism of trauma, intracranial HTN, presence of SDH, contusions, diffuse DAI, SAH, IVH, dilated pupils, FAST-positive status, decompressive craniectomy performed, and perioperative factors (blood loss, duration of surgery, blood product transfusion, colloid administration). Final outcome at discharge adjusted for primary and secondary mortality, age, mechanism of trauma, comorbidities at admission, inotropes requirement, dilated pupils, FAST-positive status, intracranial HTN, associated C-spine injury, and perioperative factors

Cl confidence interval, $P C T$ procalcitonin

with results of our study. Unlike other studies, we did not observe statistical significance on clinical parameters like sepsis, ICU stay, GOS, and secondary mortality in TBI patients with extracranial injuries. Moreover, this can be justified on basis of comparable ISS in each group of patients. However, PCT levels in septic TBI patients after admission (on day 2 and 5) were found to be significantly higher than other TBI patients overall in this study.

In our study, following severe TBI with median ISS of 24,31 of the 186 analyzed patients died within 5 days after trauma. There was a trend of significantly higher PCT levels $(\geq 6.37 \mathrm{ng} / \mathrm{ml})$ within first 3 days in nonsurvivors after TBI as compared with those who survived. All patients who died because of secondary etiology (sepsis or related complications) had PCT levels greater than $1.15 \mathrm{ng} / \mathrm{ml}$ at all times. However, polytrauma patients with lung injury were found to have relatively higher PCT levels than rest of multiple injured patients, though found to be statistically insignificant. This has been observed that serum PCT levels were significantly higher in VAP patients who did not survive when compared to survivors with VAP [19]. In pulmonary injury and pulmonary infection cases, the circulating PCT level and other precursors of calcitonin rise promptly, i.e., plausibly explained by release of sepsis-associated cytokines from pulmonary neuroendocrine cells of the bronchial epithelium and/or mononuclear cells [20,21]. Approximately
$23 \%$ among the studied patients had sepsis/bacteremia (2nd-day PCT level $\geq 1.15 \mathrm{ng} / \mathrm{ml}$ ) at any point of hospital or ICU stay; out of them just $8 \%$ died primarily from it. This is in contrast with other studies where lethal outcome incidence reported to be quite significant $[6,22]$. Jensen et al. [23] demonstrated that mortality was significantly higher when PCT level exceeded $1.0 \mathrm{ng} / \mathrm{mL}$ on 2nd day, which was nearly in consonance with our results. The low incidence of sepsis and resulted mortality in our study may be justified on the basis of standard hygienic patient care on 1:1 (patient: nurse) ratio, point of care diagnostic and measurement tools, and hospital protocol including empirical broad spectrum antibiotics in all moderate to severe TBI patients.

In TBI patients, we observed initial PCT concentration on admission with a cutoff value of $5.5 \mathrm{ng} / \mathrm{ml}$ had a sensitivity and specificity of $75 \%$ approximately (with AUC 0.78 ) for primary mortality. Patients status post-intestinal and cardiothoracic surgery have been found to have a cutoff PCT level of $1.5-2 \mathrm{ng} / \mathrm{ml}$ as a marker of poor outcome [24-26]. Other factors that may lead to rise in PCT levels are post-major surgery, cardiogenic shock, medications, severe burns, and coexisting cancer [25, 27]. Moreover, any condition that decreases the blood oxygen level, irrespective of cause, can cause an elevated PCT even without infection (e.g., asthma, pneumonia, and cardiac arrest). The etiopathogenesis of the PCT rise after trauma 
is indicated to be multifactorial. Experimental studies have demonstrated that apart from bacterial endotoxins, many cytological factors like cellular-level interactions, their time-dependent activation, and response when preexposed to proinflammatory stimuli (TNF alpha, IL-2, IL-6) also contribute to its elevation [20, 28]. Polytrauma and postoperative patients may also show a PCT-level rise, suggestive of inflammation, during the post-traumatic or early postoperative period independently [16, 17, 24]. Understanding this at a cytological level, quantitative PCT levels are produced by only adherent monocytes than the circulating monocytes since the adhesion receptors and molecules are also expressed in traumatized tissue.

However, with the given sensitivity/specificity this is uncertain to validate possible utility of PCT in TBI to predict early in-hospital mortality and this deserves to have further more trials on it. Further, extrapolating the results of this study may help in utilizing life-saving resources in severe TBI patients effectively in resourcelimited developing areas/countries. The major limitations of this study were: limited data on diversity of associated injuries in polytrauma patients, assessment of outcomes mainly in terms of mortality (GOS-1), limited serial PCT sample collection after admission (on days 2 and 5), and limited logistic support on ICP monitoring devices through the study period.

\section{Conclusion}

Our findings do not fully support given hypothesis as PCT does not predict outcome in TBI patients as accurately as the physical or clinical examination. The predicted relationship between PCT levels and outcome was not established, and that these results do not support the prognostic utility of PCT biomarker for outcome assessment in TBI patients with or without extracranial injuries.

\footnotetext{
Author details

${ }^{1}$ Department of Neuroanaesthesiology and Critical Care, Neurosciences Centre, All India Institute of Medical Sciences, New Delhi, 7th Floor, 710, New Delhi 110029, India. ${ }^{2}$ Department of Lab Medicine, J.P.N. Apex Trauma Centre, All India Institute of Medical Sciences, New Delhi, India. ${ }^{3}$ Department of Critical Care and Intensive Care, J.P.N. Apex Trauma Centre, All India Institute of Medical Sciences, New Delhi, India. ${ }^{4}$ Department of Microbiology, J.P.N. Apex Trauma Centre, All India Institute of Medical Sciences, New Delhi, India. ${ }^{5}$ Department of Neurosurgery, Neurosciences Center, All India Institute of Medical Sciences, New Delhi, India.
}

\section{Acknowledgements}

We are sincerely thankful to Dr. Ashish Datt Upadhyay, (Ph.D.) biostatistician, Department of biostatistics, AllMS, New Delhi, India, for his graceful inputs in statistical analysis and revising this manuscript.

\section{Author Contributions}

KG, GPS, HP helped in concept/idea/research design. GST, KG, SK, and KS wrote the manuscript. GST collected the data. GST, SK, KG, GPS, and KS analyzed the data. KG, GPS, HP, PM, KDS and RA helped in project management. SK, PM, and KS provided facilities/equipment. KDS and RA provided institutional liaisons. SK, GPS, KDS, RA, and HP gave consultation (including review of manuscript before submitting).

\section{Source of Support \\ None.}

\section{Conflict of interest}

The authors declare that they have no conflict of interest.

\section{Ethical Approval/Informed Consent}

This study was approved by the institutional ethics committee and written informed consent was obtained from the legal representative of the patients.

\section{Publisher's Note}

Springer Nature remains neutral with regard to jurisdictional claims in published maps and institutional affiliations.

Published online: 12 June 2020

\section{References}

1. Balci C, Sungurtekin H, Gürses E, Sungurtekin U, Kaptanoglu B. Usefulness of procalcitonin for diagnosis of sepsis in intensive care unit. Crit Care. 2003;7:85-90.

2. Maruna P, Nedelníková K, Gürlich R. Physiology and genetics of procalcitonin. Physiol Res. 2000;49(Suppl 1):S57-61.

3. Balci C, Sivaci R, Akbulut G, Karabekir HS. Procalcitonin levels as an early marker in patients with multiple trauma under intensive care. J Int Med Res. 2009;13:1709-17.

4. Becker KL, Snider R, Nylen ES. Procalcitonin in sepsis and systemic inflammation: a harmful biomarker and a therapeutic target. Br J Pharmacol. 2010;159(2):253-64.

5. Vijayan AL, Vanimaya, Ravindran S, et al. Procalcitonin: a promising diagnostic marker for sepsis and antibiotic therapy. J Intensive Care. 2017;5:51. https://doi.org/10.1186/s40560-017-0246-8.

6. Deng $\mathrm{S}$, Zhu H, Wang K, Cao T. Procalcitonin as a marker of sepsis and outcome in patients with neurotrauma: an observation study. BMC Anesthesiol. 2013;13:48.

7. Maier M, Wutzler S, Lehnert M, Szermutzky M, Wyen H, Bingold T, et al. Serum procalcitonin levels in patients with multiple injuries including visceral trauma. J Trauma. 2009;66(1):243-9.

8. Shekhar C, Gupta LN, Premsagar IC, Sinha M, Kishore J. An epidemiological study of traumatic brain injury cases in a trauma centre of New Delhi (India). J Emerg Trauma Shock. 2015;8(3):131-9.

9. Baker SP, O'Neill B, Haddon W Jr, Long WB. The Injury Severity Score: a method for describing patients with multiple injuries and evaluating emergency care. J Trauma. 1974;14:187-96.

10. Marshall LF, Marshall SB, Klauber MR, Clark MV. A new classification of head injury based on computerized tomography. J Neurosurg. 1991;75:S14-20.

11. Assicot M, Gendrel D, Carsin H, Raymond J, Guilbaud J, Bohuon C. High serum procalcitonin concentrations in patients with sepsis and infection. Lancet. 1993;341:515-8.

12. Carlet J. Rapid diagnostic methods in the detection of sepsis. Infect Dis Clin N Am. 1999;13:483-94.

13. Christ-Crain M, Stolz D, Bingisser R, Muller C, Miedinger D, Huber PR, Zimmerli W, Harbarth S, Tamm M, Muller B. Procalcitonin guidance of antibiotic therapy in community-acquired pneumonia: a randomized trial. Am J Respir Crit Care Med. 2006;174:84-93.

14. Christ-Crain M, Stolz D, Bingisser R, Gencay MM, Huber PR, Tamm M, Muller B. Effect of procalcitonin-guided treatment on antibiotic use and outcome in lower respiratory tract infections. Lancet. 2004;363:600-7. 
15. Nobre V, Stephan Harbarth S, Graf J, Rohner P, Pugin J. Use of procalcitonin to shorten antibiotic treatment duration in septic patients. Am J Respir Crit Care Med. 2007;177:498-505.

16. Meisner M, Tschaikowsky K, Hutzler A, Schick C, Schüttler J. Postoperative plasma concentrations of procalcitonin after different types of surgery. Intensive Care Med. 1998;24:680-4.

17. Wanner GA, Keel M, Steckholzer U, Beier W, Stocker R, Ertel W. Relationship between procalcitonin plasma levels and severity of injury, sepsis, organ failure, and mortality in injured patients. Crit Care Med. 2000;28:950-7.

18. Mokart D, Merlin M, Sannini A, Brun JP, Delpero JP, Houvenaeghel G, Moutardier $V$, Blache JL. Procalcitonin, interleukin- 6 and systemic inflammatory response syndrome (SIRS): early markers of postoperative sepsis after major surgery. Br J Anaesth. 2005:94:767-73.

19. Duflo F, Debon R, Monneret G, Bienvenu J, Chassard D, Allaouchiche B. Alveolar and serum procalcitonin: diagnostic and prognostic value in ventilator-associated pneumonia. Anesthesiology. 2002;96:7479.

20. Oberhoffer M, Stonans I, Russwurm S, Stonane E, Vogelsang H, Junker U, Jaeger $L$, Reinhart K. Procalcitonin expression in human peripheral blood mononuclear cells and its modulation by lipopolysaccharides and sepsis related cytokines in vitro. J Lab Clin Med. 1999;134:49-55.

21. Nylen ES, Snider RH Jr, Thompson KA, Rohatgi P, Becker KL. Pneumonitisassociated hyperprocalcitoninemia. Am J Med Sci. 1996;312:1218.
22. Luyt CE, Guérin V, Combes A, Trouillet JL, Ayed SB, Bernard M, Gibert C, Chastre J. Procalcitonin kinetics as a prognostic marker of ventilatorassociated pneumonia. Am J Respir Crit Care Med. 2005;171:48-53.

23. Uzzan B, Cohen R, Nicholas P, Cucherat M, Perret GY. Procalcitonin as a diagnostic test for sepsis in critically ill adults and after surgery or trauma: a systematic review and meta-analysis. Crit Care Med. 2006;34:1996-2003.

24. Meisner M, Rauschmayer C, Schmidt J, Feyrer R, Cesnjevar R, Bredle D, Tschaikowsky K. Early increase of procalcitonin after cardiovascular surgery in patients with postoperative complications. Intensive Care Med. 2002;28:1094-102.

25. Reith HB, Mittelkötter U, Debus ES, Kussner C, Thiede A. Procalcitonin in early detection of postoperative complications. Dig Surg. 1998;15:260-5.

26. Müller B, Becker KL, Schachinger H, Rickenbacher PR, Huber PR, Zimmerli W, Ritz R. Calcitonin precursors are reliable markers of sepsis in a medical intensive care unit. Crit Care Med. 2000;28:977-83.

27. Samsudin I, Vasikaran SD. Clinical utility and measurement of procalcitonin. Clin Biochem Rev. 2017;38(2):59-68.

28. Nijsten MW, Olinga P, The TH, de Vries EG, Koops HS, Groothuis GM, Limburg PC, ten Duis HJ, Moshage H, Hoekstra HJ, Bijzet J, Zwaveling JH. Procalcitonin behaves as a fast responding acute phase protein in vivo and in vitro. Crit Care Med. 2000;28(2):458-61. 